\title{
Barrier effects on the collective excitations of split Bose-Einstein condensates
}

\author{
P. Capuzzi and E. S. Hernández \\ Departamento de Física, Facultad de Ciencias Exactas y Naturales, \\ Universidad de Buenos Aires, RA-1428 Buenos Aires, \\ ${ }^{1}$ and Consejo Nacional de Investigaciones Científicas y Técnicas, Argentina
}

\begin{abstract}
We investigate the collective excitations of a single-species Bose gas at $T=0$ in a harmonic trap where the confinement undergoes some splitting along one spatial direction. We mostly consider onedimensional potentials consisting of two harmonic wells separated a distance $2 z_{0}$, since they essentially contain all the barrier effects that one may visualize in the 3D situation. We find, within a hydrodynamic approximation, that regardless the dimensionality of the system, pairs of levels in the excitation spectrum, corresponding to neighbouring even and odd excitations, merge together as one increases the barrier height up to the current value of the chemical potential. The excitation spectra computed in the hydrodynamical or Thomas-Fermi limit are compared with the results of exactly solving the time-dependent Gross-Pitaevskii equation. We analyze as well the characteristics of the spatial pattern of excitations of threedimensional boson systems according to the amount of splitting of the condensate.
\end{abstract}

Pacs 03.75.Fi,05.30.Jp,32.80.Pj

\section{INTRODUCTION}

The experimental realization of Bose-Einstein condensation (BEC) [1] 3] has inspired an impressive amount of further laboratory research together with important theoretical advances. The overwhelming material produced in the last three years has been recently reviewed by several authors [4 [6]. Among the various fascinating subjects that have attracted the attention of the community, the predictions of the spectrum of low-lying excitations and sound velocity [7,8] in a hydrodynamic picture based on perturbations over the Thomas-Fermi regime, and their experimental verification [9 [12] play a special role, since the excellent agreement between theoretical and observed figures bring into evidence the validity of the mean field description of large condensates. Methods to obtain exact solutions of the time-dependent Gross-Pitaevskii equation (GPE) have been developed [13] which permit further confirmation of the accuracy of the TF+hydrodynamic approach in the thermodynamic limit; moreover, the large amplitude motion of a condensate has been investigated in a fashion that demonstrates the validity of a scaling hypothesis [14], according 
to which a condensate may evolve mantaining the appearance of a barometric distribution in a quadratic field.

A particular subject of the BEC field is the possibility of forming and studying double condensates from several viewpoints. Starting from the original MIT trap [2] and its later improvements that allowed, for instance, the observation of interference fringes and the experimental realization of the coherence of matter waves [15], the isomorfism between atomic condensates and optical devices has been intensively investigated [16]. Tunneling between the wells and the features of Josephson currents now constitute common subjects in the literature [17,[18]. A special chapter includes the binary mixtures of condensates or double condensates of distinguishable particles, typically associated to hyperfine splitting of the magnetic atom levels in the trap [19]. In particular, a mean field description of two species, spatially split double condensates has been advanced in Ref. [23]. Indeed, most theoretical approaches regarding the low energy dynamics of a double well [20,21, tunneling and/or Josephson effects consider essentially this type of distinguishable systems - or concentrate upon dynamical aspects that exclude the quantum and thermodynamic analysis of the double well as a subject in its own right. The investigation of the properties of single-species, double condensates with substantial spatial overlapping could, however, become relevant in the near future in view of the current possibility of adiabatically distorting traps [22] creating long-lived systems with various minima and barriers [32].

Recently, we have advanced some results on the thermodynamics of BEC of indistiguishable particles in harmonic double wells [24], which indicate that with a minimum amount of complication with respect to the well-known harmonic traps, one can trace the evolution of the atomic spectrum as a single potential becomes progressively split into two with the same frequency, at a distance $2 z_{0}$ apart along the $z$-axis, and separated by a barrier $V_{0}=M \omega_{z}^{2} z_{0}^{2} / 2$. The major features of BEC, under such conditions, can be accurately described and interpreted in terms of a smooth transition from a system with $N$ particles to two identical ones with $N / 2$ each. With this in mind, in the present work we intend to analyze the effects of the presence of such barriers upon the collective excitations of the condensate. For this sake, in Sec. 2 we concentrate on the onedimensional, harmonic double well problem; we extract the exact eigenspectrum of the linearized hydrodynamical equation [7], and confront the results thus obtained with the exact Fourier spectrum of the GPE solutions. In Sec. 3, we examine the threedimensional system and examine, in particular, the spatial patterns of the perturbation as the well splitting increases. Section 4 is devoted to the full discussion of the results here presented. The final summary and comments are the subject of Sec. 5 .

\section{ONEDIMENSIONAL BOSE SYSTEM IN A HARMONIC DOUBLE WELL}

The general formalism leading from the time dependent Gross-Pitaevskii equation (GPE) to the description of the low energy excitations of a Bose-Einstein condensate has been derived by Stringari [7] and reviewed, for example, in Refs. [4,6]. The procedure consists of linearizing the hydrodynamical version of the GPE with respects to small deviations $\delta \rho$ from the Thomas-Fermi equilibrium density inside the condensate

$$
\rho_{T F}(\mathbf{r})=\frac{\mu-V_{e x t}(\mathbf{r})}{g}
$$


where $\mu$ is the chemical potential evaluated in the Thomas-Fermi regime and $g=4 \pi \hbar^{2} a / M$ is the hard-sphere interaction strength for an $s$-wave scattering length $a$. This linearized equation reads

$$
M \Omega^{2} \delta \rho=-\nabla \cdot\left[\left(\mu-V_{e x t}\right) \nabla \delta \rho\right]
$$

Its one dimensional version, namely

$$
M \Omega^{2} \delta \rho=-\frac{\partial}{\partial z}\left[\left(\mu-V_{e x t}(z)\right) \frac{\partial}{\partial z} \delta \rho\right]
$$

with $V_{\text {ext }}$ a harmonic potential with frequency $\omega$, after an adequate scaling is precisely the differential equation satisfied by the Legendre polynomials $P_{n}\left(\sqrt{M \omega^{2} / 2 \mu_{0}} z\right)$, the corresponding eigenvalues being of the form $\Omega_{n}^{2}=\omega^{2} n(n+1) / 2$ [26]. Furthermore, the TF chemical potential $\mu_{0}$ reads, in this case,

$$
\mu_{0}=\frac{\hbar \omega}{2}\left(6 \pi N \frac{a}{a_{z}}\right)^{2 / 3}
$$

with $a_{z}=\sqrt{\hbar / M \omega}$ the corresponding oscillator length.

Our current purpose is to find the exact solutions of Eq. (2.3) when $V_{\text {ext }}$ is the double well potential

$$
V_{\text {ext }}=\frac{1}{2} M \omega^{2}\left(|z|-z_{0}\right)^{2}
$$

In this case, within the TF approximation, the ratio of the chemical potential $\mu$ to that of a single well is found to satisfy the relationships

$$
\begin{aligned}
& \frac{\mu}{\mu_{0}}=\frac{1}{2^{2 / 3}} \text { if } V_{0} \geq \mu \\
& \left(\frac{\mu}{\mu_{0}}\right)^{3 / 2}+\frac{3}{4}\left(\frac{V_{0}}{\mu_{0}}\right)^{1 / 2}\left(\frac{\mu}{\mu_{0}}\right)-\frac{1}{2}\left(\frac{V_{0}}{\mu_{0}}\right)^{3 / 2}=1 \text { otherwise }
\end{aligned}
$$

where the barrier effect is fully included in the ratio $V_{0} / \mu_{0}$. The decrease $\mu / \mu_{0}$ of the chemical potential is plotted as a function of the dimensionless variable $\sqrt{V_{0} / \mu_{0}}=z_{0} / Z_{0}$, with $Z_{0}$ the single-condensate size, in Fig. 1, while in Fig. 2 we display three TF densities, respectively corresponding to $\mu=5 / 2 V_{0}, V_{0}$ and $1 / 4 V_{0}$.

We now look for the solutions of Eq. (2.3) in a fashion that closely parallels the computation of the eigenvalues of Schrödinger equation for the double well hamiltonian [25]. In other words, we look for eigenvalues $\Omega_{\nu}^{2}$ and eigenvectors $\delta \rho_{\nu}$ of the form

$$
\Omega_{\nu}^{2}=\frac{\omega^{2}}{2} \nu(\nu+1)
$$

and 


$$
\delta \rho_{\nu}=P_{\nu}\left[\sqrt{M \omega^{2} / 2 \mu}\left(|z|-z_{0}\right)\right]
$$

with $P_{\nu}(x)$ a generalized Legendre function [27]. To obtain the complete solution of the hydrodynamic problem we observe that i) the operator on the right-hand side of (2.3) has well defined parity and ii) the boundary condition that guarantees real values of $\Omega$ is the nondivergence of the density fluctuation on the edges of the condensate [28]. Taking this into account, we construct the eigensolutions (2.8) separately for positive and negative $z$ and request the matching conditions at $z=0$ [25],

$$
\begin{gathered}
P_{\nu}\left[-\sqrt{M \omega^{2} / 2 \mu} z_{0}\right]=0 \text { for odd solutions, } \\
P_{\nu}^{\prime}\left[-\sqrt{M \omega^{2} / 2 \mu} z_{0}\right]=0 \text { for even solutions. }
\end{gathered}
$$

When the barrier height reaches the current chemical potential value $\mu$, Eq. (2.3) is well defined in two separate domains (cf. Fig. 2) and we can construct the density fluctuations as even and odd superpositions of the exact single-well solutions.

$$
\begin{aligned}
\delta \rho_{\text {even }} & =\mathcal{N}\left\{\delta \rho_{0}\left(z-z_{0}\right) \Theta(z)+\delta \rho_{0}\left(-z-z_{0}\right) \Theta(-z)\right\} \\
\delta \rho_{\text {odd }} & =\mathcal{N}\left\{\delta \rho_{0}\left(z-z_{0}\right) \Theta(z)-\delta \rho_{0}\left(-z-z_{0}\right) \Theta(-z)\right\}
\end{aligned}
$$

being $\delta \rho_{0}$ one eigensolution for the 1D harmonic trap with a length scale corresponding to halving the particle number, and $\Theta(x)$ the Heavyside function. Thus, once the condensate is split into two pieces, under the TF approximation, the excitation spectrum for any finite barrier $V_{0}$ higher than the ground state energy $\mu$ is the single well one, however doubly degenerate. The appearance of the 1D excitation spectrum as a function of the separation $\sqrt{V_{0} / \mu}=z_{0} / Z$ is depicted in Fig. 3; moreover, to inspect the departures in the behavior of the excitations from the hydrodynamic limit, we have integrated the time dependent GP equation and display with symbols some indicative results. This integration is achieved in two steps: we first build up the condensate wavefunction solving the time-independent problem by the steepest descent method [30], and afterwards we add some noise to the condensate density $\rho_{0}$. We then follow the time evolution of the perturbed density and compute the fast Fourier transform (fft) at one minimum of the double well potential, which provides the spectrum of small amplitude oscillations of the double condensate [31. This is illustrated in Fig. 4, where we plot this spectrum for four choices of the ratio $\sqrt{V_{0} / \mu}$ and an interaction strength $N a / a_{z}=10$. We realize that for very low barriers, the spectrum is the one corresponding to a single well, but as one increases the separation between the minima, two quasidegenerate branches can be distinguished. These branches completely merge at infinitely large barriers, however, they remain observable at finite values of $V_{0}$ due to the limited resolution on the fft calculation. We notice that although the slopes of the odd eigenvalues of the TF hydrodynamics exhibit a sharp discontinuity at the barrier limit $V_{0} / \mu=1$, the fft results behave smoothly across the transition. We shall analyze this issue later in Sec. 4. 


\section{THE THREEDIMENSIONAL BOSE SYSTEM}

We now discuss the solution of the full equation (2.2) for a cylindrical potential of the form

$$
V_{\text {ext }}(\mathbf{r})=\frac{M \omega_{r}^{2}}{2} r^{2}+\frac{M \omega_{z}^{2}}{2}\left(|z|-z_{0}\right)^{2}
$$

with a given aspect ratio $\lambda=\omega_{z} / \omega_{r}$. In this case, the chemical potential $\mu$ in the ThomasFermi limit is related to the single-well one given by

$$
\mu_{0}=\frac{\hbar \tilde{\omega}}{2}\left(15 N \frac{a}{\tilde{a}}\right)^{2 / 5}
$$

with $\tilde{\omega}=\left(\omega_{r}^{2} \omega_{z}\right)^{\frac{1}{3}}$ and $\tilde{a}$ the corresponding oscillator length, according to

$$
\begin{aligned}
& \frac{\mu}{\mu_{0}}=\frac{1}{2^{2 / 5}} \text { if } V_{0}>\mu \\
& \left(\frac{\mu}{\mu_{0}}\right)^{5 / 2}+\frac{15}{8}\left(\frac{V_{0}}{\mu_{0}}\right)^{1 / 2}\left(\frac{\mu}{\mu_{0}}\right)^{2}-\frac{5}{4}\left(\frac{V_{0}}{\mu_{0}}\right)^{3 / 2}\left(\frac{\mu}{\mu_{0}}\right)+\frac{3}{8}\left(\frac{V_{0}}{\mu_{0}}\right)^{5 / 2}=1 \text { otherwise }
\end{aligned}
$$

The ratio $\mu / \mu_{0}$ is displayed in Fig. 5 as a function of the dimensionless variable $\sqrt{V_{0} / \mu_{0}}$ and in Fig. 6, we display three contour plots of a TF density - i.e., the condensate edge and two inner equiprobability contours - for a number of particles such that $\mu=5 / 2 V_{0}$. Alternatively, these curves represent three different condensate edges for $\mu=5 / 2 V_{0}, V_{0}$ and $1 / 4 V_{0}$, in correspondence with the $1 \mathrm{D}$ density profiles of Fig. 2.

Due to the different definition of the $z$-components of the restoring force for positive and negative values of the coordinate, standard methods [7,8, 28,29] employed to derive the eigenfunctions and eigenvalues for anisotropic single-well potentials are not especially useful in this case. We have rather found convenient to expand the solutions $\delta \rho(r, z)$ in the spherical basis $\left.P^{(2 n)}(r) r^{l} Y_{l m}(\theta, \phi)\right)$ [7], and diagonalize the matrix representing the operator $\nabla \cdot\left[\left(V_{\text {ext }}-\mu\right) \nabla\right] / M$. The eigenspectra thus obtained are labelled by the magnetic quantum number $m$ and in order to illustrate the appearance of the spectra $\Omega_{m k}$, with $k$ just numbering the level for the given $m$, in Fig. 7 we display these energies for (a) $m=0$ and (b) $m=1$ as functions of the well separation. We appreciate the general trend similar to that already analyzed in the 1D case, namely, the gradual merge of pairs of levels corresponding to even and odd eigenfunctions and their collapse into the doubly degenerate spectrum of singlewell excitations. The apparent discontinuity of the odd level energies when the chemical potential equals the barrier height is also visible; furthermore, the eigenfrequencies arising from the numerical diagonalization can be compared with those provided by the Fourier transformation of an initial perturbation that evolves according to the full GPE dynamics. Figure 8 displays a typical Fourier spectrum for a weak barrier with $\sqrt{V_{0} / \mu}=0.14$; some eigenvalues of the dynamical matrix are indicated on the abscissa axis so as to remark the good agreement between both procedures, in spite of the inaccuracies intrinsic to the Thomas-Fermi approximation. However, the way in which the excitation was created in the $\mathrm{fft}$ method - 1.e, adding some random fluctuation to the TF ground state - does not 
allow us to control exactly the particular selection of the fluctuating modes or their relative intensity. Thus, there exist eigenfrequencies of the problem that we cannot clearly see in the fft spectrum of an arbitrary initial condition like the typical example in Fig. 8.

The shape of the perturbation $\delta \rho_{m k}(r, z)$ as a function of the coordinates inside the condensate deserves some comments. We have chosen to analyze the transition density patterns in the following way: keeping Fig. 6 as a reference, we consider, for a given condensate characterized by a choice of the chemical potential $\mu$, the equiprobability contours $\rho_{T F}(r, z)=\rho_{0}$ with $\rho_{0}$ a constant that vanishes at the condensate edge and increases towards the center of the trap. In Fig. 9, we are drawing four portraits corresponding to the first odd parity excitation for $m=0$, for different well separations (or to different chemical potentials for fixed barrier height). Each of these sets contains curves $\delta \rho[r(z), z]$ as functions of $z$ for a selected equiprobability contour $\rho_{0}$. Full lines indicate the condensate edge $\rho_{0}=0$, while the different dashed lines correspond to inner contours of increasing equal density. Figures 10 to 12 respectively display the same patterns for the first even parity excitation with $m=0$ and for the first odd and first even parity eigenmodes with $m=1$. Very similar scenarios are obtained for other low energy levels, the present choice being convenient to observe the evolution of the perturbation and its nodal characteristics in terms of relatively simple, however rich enough, spatial structure. We examine these results further in Sec. 4.

\section{DISCUSSION OF THE RESULTS}

From the previous chapters, we recognize that the most important differences between the present collective dynamics and the well-known excitation spectra of Bose condensates in anisotropic harmonic wells, can be fully understood in terms of the dynamical properties of the $1 \mathrm{D}$ double well. It is interesting to remark that, in this situation, the hydrodynamical wave equation (2.3) can be solved almost analytically in the same spirit, and with the same degree of complexity, as the quantum problem of the Schrödinger eigenspectrum of a harmonic double well. The behavior of the excitation energies under a progressive well splitting (cf. Figs. 3 and 6) is a monotonic decrease together with an evolution towards doubly degeneracy of the excitations of a single harmonic well. This can be understood in terms of a simple classical picture that illustrates the effect of the barrier; in fact, the hydrodynamic equation (2.3) may be interpreted as the classical wave equation of a perturbing density fluc-

tuation $\delta \rho$ travelling inside the condensate with a local velocity $v(z)=\sqrt{\left(\mu-V_{\text {ext }}(z)\right) / M}$. With this in mind, we can estimate the time $\tau$ employed by the perturbation to go from one well to the other and the oscillation period $T$, namely,

$$
\begin{aligned}
\tau & =\int_{-a}^{a} d z \frac{1}{v(z)}=\frac{2 \sqrt{2}}{\omega} \operatorname{Arcsin}\left(\frac{V_{0}}{\mu}\right) \\
T & =2 \int_{-a-Z}^{a+Z} d z \frac{1}{v(z)}=\frac{2 \pi \sqrt{2}}{\omega}\left[1+\frac{2}{\pi} \operatorname{Arcsin}\left(\frac{V_{0}}{\mu}\right)\right] \\
& =T_{0}+2 \tau
\end{aligned}
$$

being $T_{0}$ the period of the orbit of energy $\mu$ for a particle with mass $2 M$ in a single well of frequency $\omega$; one may notice that under the replacement $p=M \omega r$, Fig. 6 describes as well the classical phase portrait of a particle in the 1D-double well. We realize then that when 
$V_{0}$ is sufficiently low, the fluctuation essentially oscillates around the condensate along the classical orbit with period $T_{0}$; in particular, when $V_{0}$ equals $\mu$, the excitation requires twice a period to cross the double condensate, the classical orbit -pictures in medium dashes in Fig. 6 - being the separatrix between the two different oscillation regimes. As $V_{0}$ grows up, a delay equal to $2 \tau$ appears in the oscillation time, reflecting the finite amount of time requested to switch back and forth between the two wells.

The decrease of the eigenfrequencies can thus be unambiguously interpreted in terms of the enlarging of the classical period of the orbits due to the presence of a finite barrier. However, the most striking outcome of the TF computation is the discontinuity of the odd-state engenfrequencies as we approach the limiting regime $\mu-V_{0}$ from above. In fact, contrasting this behavior with the strict Fourier spectrum, for which some results are indicated with symbols in Fig. 3, we realize that this discontinuity is an artifact of the TF approach, removable by an exact calculation. The explanation is as follows: let us project the wave equation onto an eigenstate, thus expressing the eigenvalues in the form

$$
\Omega_{\nu}^{2}=\frac{1}{M} \int d z\left|\frac{d \delta \rho_{\nu}}{d z}\right|^{2}\left[\mu-V_{e x t}(\mathbf{r})\right]
$$

where the integration is to be performed within the borders of the TF condensate. If $\mu$ is lower than the barrier $V_{0}$, the condensate is split into two disjoint condensates and the region around $z=0$ is excluded from the integration domain. This exclusion is not significant for even parity states, since the density gradient vanishes at $z=0$; however, this is not the case for the odd parity levels, whose exact Fourier spectrum, lying higher than the TF one (cf. Fig. 3) includes the contributions to the eigenfrequencies which stem from the whole spatial domain, in particular, the subbarrier region where the exact Gross-Pitaevskii wave function yields finite probability densities for the trapped bosons.

This issue can be also easily interpreted taking into account that if one excites the condensate, generating a small density fluctuation with even parity, this excitation will oscillate in time within the limits of each well, basically unaffected by the presence of the other. By contrast, if the fluctuation is an odd parity one, a peak in one side of the condensate is imaged by a dip in the other; subsequent time evolution forces this pattern to oscillate from one side to the other, crossing the barrier. Now, since within the TF approximation both the condensate and its fluctuations are bounded to the region $V_{e x t}<\mu$, we recognize that when the barrier increases above the chemical potential value, any pertubation is forced to remain on its side with no posible interwell transition - thus the levels correspond to two independent Bose-Einstein condensates-. This is not what happens in a real double well described by the full GPE, where both condensates interpenetrate.

The 3D situation gives us the opportunity to investigate transition density profiles for different selections of labels and for several possible manners of disecting the condensate. In Sec. 3 we have displayed some of the spatial characteristics of the first odd and even $m=0$ and 1 modes (in Figs. 9 to 12), choosing the variable $z$ to parametrize various equiprobability contours inside the condensate. The evolution of these patterns illustrates several interesting facts. We first realize that the spatial distribution of the perturbation evolves, with increasing well separation, so as to eliminate nodes of the eigenfunction, in order to account for the level merging visible in Figs. 3 and 7. Secondly, we observe the transformation from a pattern localized in one harmonic potential to a pattern of the same nature, however 
split into the two disjoint wells. In particular, the fourth portrait of each of these figures corresponds to an almost split condensate. We notice that in neither case the exact form of the twofold distribution after separation coincides with the original one, due to the fact that in the large $V_{0}$ limit, the fluctuation is of the form (2.11) with the parity of $\delta \rho_{0}$ uncorrelated to that of the starting $\delta \rho$. Thus, should one duplicate the original pattern in each well after their definitive splitting, the energy would be higher. We observe that the $m=0$ modes display finite density fluctuations in the outer edges of the condensate $\left(z= \pm\left(z_{0}+Z\right)\right)$ whereas in $z=0$, where the inner boundary surface grows up with increasing well separation, the density vanishes or not according to the parity being with odd or even; by contrast, in either case the $m=1$ modes intend to concentrate the fluctuations at values of $z$ far from either the outer (i.e., $\left.z \approx \pm\left(z_{0}+Z\right)\right)$ or the inner $(z \approx 0)$ condensate edges. The effect of the barrier upon even parity perturbations is to introduce a discontinuity in the slope of $\delta \rho$, similar to that in the TF density itself. We also see that, as in the single-well problem, the inner contours, where the above density is larger, experience weaker density fluctuations than the condensate edge.

\section{SUMMARY}

In this work we have discussed the characteristics of the small amplitude collective excitations of a Bose condensed gas in harmonic wells where the confinement has undergone some splitting along one of the three directions. The excitations of the confined bosons has been investigated within the hydrodynamic limit of the GPE (TF limit), in other words, we have analyzed the small fluctuations of the condensate density in the limit of large number of particles. This enables us to determine the chemical potential as a function of the barrier height and the single-well parameters in the 1D and 3D problems. We have considered extensively the 1D potential consisting of two harmonic wells separated a distance $2 a$, since it contains essentially all the barrier effects that one may visualize later in the 3D situation.

The general results can be summarized as follows. We find that the energy levels corresponding to the density fluctuations gradually merge into pairs as one increases the barrier height; within the TF approximation, as soon as the barrier overcomes the current value of the chemical potential the spectrum becomes completely degenerate. We also have shown that the behavior of the eigenspectrum as a function of the well separation can be interpreted, in the 1D case, by means of a classical picture, taking into consideration the way in which the finite barrier affects the period of the classical orbit. The appearance of degeneracy in the eigenspectrum, that images the well-known property of the Schrödinger eigenspectrum of a 1D harmonic double potential, gives rise to various consequences upon the spatial pattern of the excitation near the surface of a 3D condensate when the latter has undergone splitting. In the 3D problem we have displayed typical excitations corresponding to levels with and without z-proyection of the angular momentum and have discussed the consequences of progressively splitting the condensate. We recognize the usual differences between fluctuations with zero and nonzero magnetic quantum number; while the first ones remain finite at the edges $z= \pm\left(z_{0}+Z\right.$ ) (cf. Figs. 9 and 10), they vanish at those positions if $m \neq 0$. These features remain valid as one increases the barrier height and splits the condensate into two pieces.

To summarize, we remark that the collective excitations of this bistable system can be 
examined on almost identical grounds as in the case of a single-well one, with an additional parameter, namely the ratio between barrier height and chemical potential, which ranges from a single-well problem for negligibles barriers to the double degenerate regime where one exactly halves the condensate into two pieces, in the TF limit of the GPE for infinite separations.

\section{ACKNOWLEDGMENTS}

We ackowledge fruitful discussions with Dr. Artur Polls. This paper was performed under grants PICT 1706 from Agencia Nacional de Promoción Científica y Tecnológica and PIP 4209/96 from Consejo Nacional de Investigaciones Científicas y Técnicas of Argentina. 


\section{REFERENCES}

[1] M. H. Anderson, J. R. Enscher, M. R. Matthews, C. E. Wieman and E. A. Cornell, Science 269, 198 (1995).

[2] K. B. Davies, M. O. Mewes, M. R. Andrews, N. J. van Druten, D. S. Durfeee, D. M. Kurn and W. Ketterle, Phys. Rev. Lett 75, 3969 (1995).

[3] C. C. Bradley, C. A. Sackett, J. J. Tollet and R. G. Hulet, Phys. Rev. Lett. 75, 1687 (1995).

[4] A. S. Parkins and D. F. Walls, Phys. Rep. 303, 1 (1998).

[5] Hua Shi and A. Griffin, Phys. Rep. 304, 1 (1998).

[6] F. Dalfovo, S. Giorgini, L. P. Pitaevskii and S. Stringari, cond-mat/9806038 (1998).

[7] S. Stringari, Phys. Rev. Lett. 77, 2360 (1996).

[8] S. Stringari, Phys. Rev. A 58, 2385 (1998).

[9] D. S. Jin, J. R. Enscher, M. R. Matthews, C. E. Wieman and E. A. Cornell, Phys. Rev. Lett. 77, 420 (1996).

[10] M. O. Mewes, M. R. Andrews, N. J. van Druten, D. M. Kurn, D. S. Durfee, C. G. Townsend and W. Ketterle, Phys. Rev. Lett. 77, 988 (1996).

[11] D. S. Jin, M. R. Matthews, J. R. Ensher, C. E. Wieman and E. A. Cornell, Phys. Rev. Lett. 78, 764 (1997).

[12] M. R. Andrews, D. M. Kurn, H. J. Miesner, D. S. Durfee, C. G. Townsend, S. Inouye and W. Ketterle, Phys. Rev. 79, 553 (1997).

[13] P. A. Ruprecht, M. J. Holland, K. Burnett and M. Edwards, Phys. Rev. A 51, 4704 (1995); M. Holland and J. Cooper, Phys. Rev. A 53, R1954 (1996); see also Ref. [30].

[14] Y. Kagan, E. L. Surkov and G. V. Shlyapnikov, Phys. Rev. A 54, R1753 (1996); Y. Castin and R. Dum, Phys. Rev. Lett. 77, 5315 (1996); Y. Kagan, E. L. Surkov and G. V. Shlyapnikov, Phys. Rev. A 55, R18 (1997).

[15] M. R. Andrews, C. G. Townsend, H. -J. Miesner, D. S. Durfee, D. M. Kurn and W. Ketterle, Science 275, 637 (1997); W. Ketterle and H. -J. Miesner, Phys. Rev. A 56, 3291 (1997); E. A. Burt, R. W. Ghrist, C. J. Myatt, M. J. Holland, E. A. Cornell and C. E. Wieman, Phys. Rev. Lett. 79, 337 (1997).

[16] E. V. Goldstein, M. G. Moore, O. Zobay and P. Meystre, cond-mat/9807392 (1998); see also chapters 4,5 and 7 of Ref. [4].

[17] H. M. Jack, M. J. Collett and D.F. Walls, Phys. Rev. A 54, R4625 (1996).

[18] J. Javanainen, Phys. Lett. A 161, 207 (1991); G. J. Milburn, J. Corney, E. Wright and D. F. Walls, Phys. Rev. A 55, 4318 (1997); J. Williams, R. Walser, J. Cooper, E. Cornell and M. Holland, cond-mat/9806337 (1998); F. Sols, cond-mat/9810338 (1998); see also ref 30.

[19] C. J. Myatt, E. A. Burt, R. W. Ghrist, E. A. Cornell and C. E. Wieman, Phys. Rev. Lett. 78, 586 (1997).

[20] E. V. Goldstein and P. Meystre, Phys. Rev. A 55, 2935 (1997).

[21] R. Graham and D. F. Walls, Phys. Rev. A 57, 484 (1998).

[22] S. M. Stamper-Kurn, H. J. Miesner, A. P. Chikkatur, S. Inouye, J. Stenger and W. Ketterle, cond-mat/9805022 (1998).

[23] B. D. Esry, Chris H. Greene, J. P. Burke, Jr. and J. L. Bohn, Phys. Lett. 78, 3594 (1997).

[24] P. Capuzzi and E. S. Hernández, Phys. Rev A, in press. 
[25] E. Merzbacher, Quantum Mechanics, Wiley, N. Y., 1961.

[26] Tin-Lun Ho and M. Ma, cond-mat/9703034 (1997)

[27] I. S. Gradstein and I. M. Rhyzhik, Table of Integrals, Series and Products, Academic Press, San Diego, 1994.

[28] M. Fliesser, A. Csordás, P. Szépfalusy and R. Graham, Phys. Rev. A 56, R2533 (1997).

[29] A. Csordás and R. Graham, cond-mat/9809002 (1998).

[30] F. Dalfovo and S. Stringari, Phys. Rev. A. 53, 2477 (1996).

[31] M. Edwards, P. A. Ruprecht, K. Burnett, R. J. Dodd and C. W. Clark, Phys. Rev. Lett. 77, 1671 (1996); P. A. Ruprecht, M. Edwards, K. Burnett and C. W. Clark, Phys. Rev. A 54, 4178 (1996).

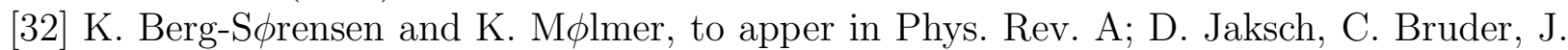
I. Cirar, C. W. Gardiner and P. Zoller, cond-mat/9805037 (1998); K-P. Marzlin and W. Zhang, cond-mat/9810085 (1998); M. J. Steel and W. Zhang, cond-mat/9810284 (1998).

\section{FIGURE CAPTIONS}

FIG. 1. The chemical potential $\mu$ for the $1 \mathrm{D}$ harmonic double well in the TF limit in units of the single well value $\mu_{0}$ as a function of the dimensionless well separation (in units of the size of the single-well condensate). The horizontal and vertical thin lines respectively indicate the chemical potential and barrier height corresponding to full splitting into two disjoint single wells.

FIG. 2. Three TF density profiles (in arbitrary units). Full lines indicate the value of the chemical potential for which the given contour is a condensate edge. The density profiles have been moved up to the corresponding chemical potential for easier visualization.

FIG. 3. Excitation spectrum (in units of $\omega$ ) of the $1 \mathrm{D}$ hydrodynamic equation (2.3) as a function of the dimensionless parameter $\sqrt{V_{0} / \mu}$. The horizontal thin lines indicate the spectrum for the single well problem. Some eigenvales provided by the fft calculation are displayed with symbols.

FIG. 4. The fast Fourier transform of the evolution of a perturbing density in the 1D-TF limit, for $\sqrt{V_{0} / \mu}=0,0.4,1.09$ and 3.9 (from top to bottom). The intensity of the peaks is displayed in arbitrary units and $\Omega$ is given in units of $\omega$.

FIG. 5. Same as Fig. 1 for the 3D double well problem.

FIG. 6. Three equiprobability contours (in arbitrary units) for a TF condensate, or equivalently, the condensate edges for the same chemical potentials considered in Fig. 2.

FIG. 7. Same as Fig. 3 for the 3D hydrodynamic problem: (a) $m=0$ and (b) $m=1$. Thin lines correspond to the single-well spectrum for the same parameters. 
FIG. 8. Spectrum of the $3 \mathrm{D}$ problem provided by the fft calculation for $\sqrt{V_{0} / \mu}=0.14$ as a function of $\Omega$ (in units of $\omega$ ). The vertical lines show a few hydrodynamic eigenfrequencies obtained through diagonalization of (2.2). The height of the peaks is set in arbitrary units.

FIG. 9. The first odd-parity density fluctuation $\delta \rho[r(z), z]$ of the condensate as a function of $z$ (in arbitrary units), for $m=0$. The four portraits show the evolution from a single well problem to the limiting case $V_{0} \sim \mu$ with the values $\sqrt{V_{0} / \mu}=0.01,0.26,0.43$ and 0.99. Each curve on each portrait corresponds to the contour values $\rho_{0} / \rho_{\max }=0$, $1 / 4,1 / 2,3 / 4$; the full lines denoting the condensate edge and $\pm\left(z_{0}+Z\right)$ points on the z-axis.

FIG. 10. Same as Fig. 9 for the first even parity eigenstate.

FIG. 11. Same as Fig. 9 for $m=1$.

FIG. 12. Same as Fig. 10 for $m=1$. 


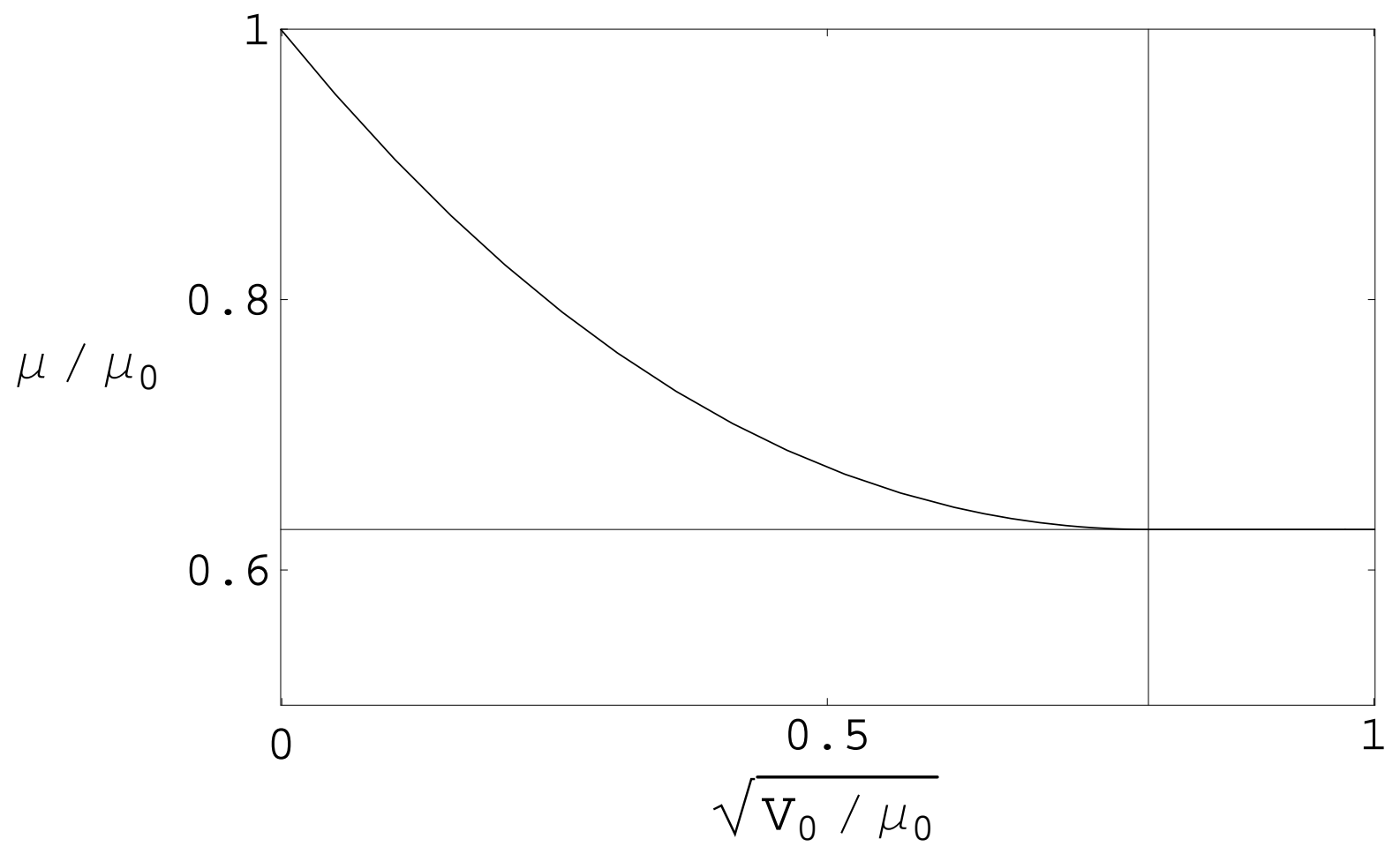

Figure 1. Capuzzi \& Hernandez 


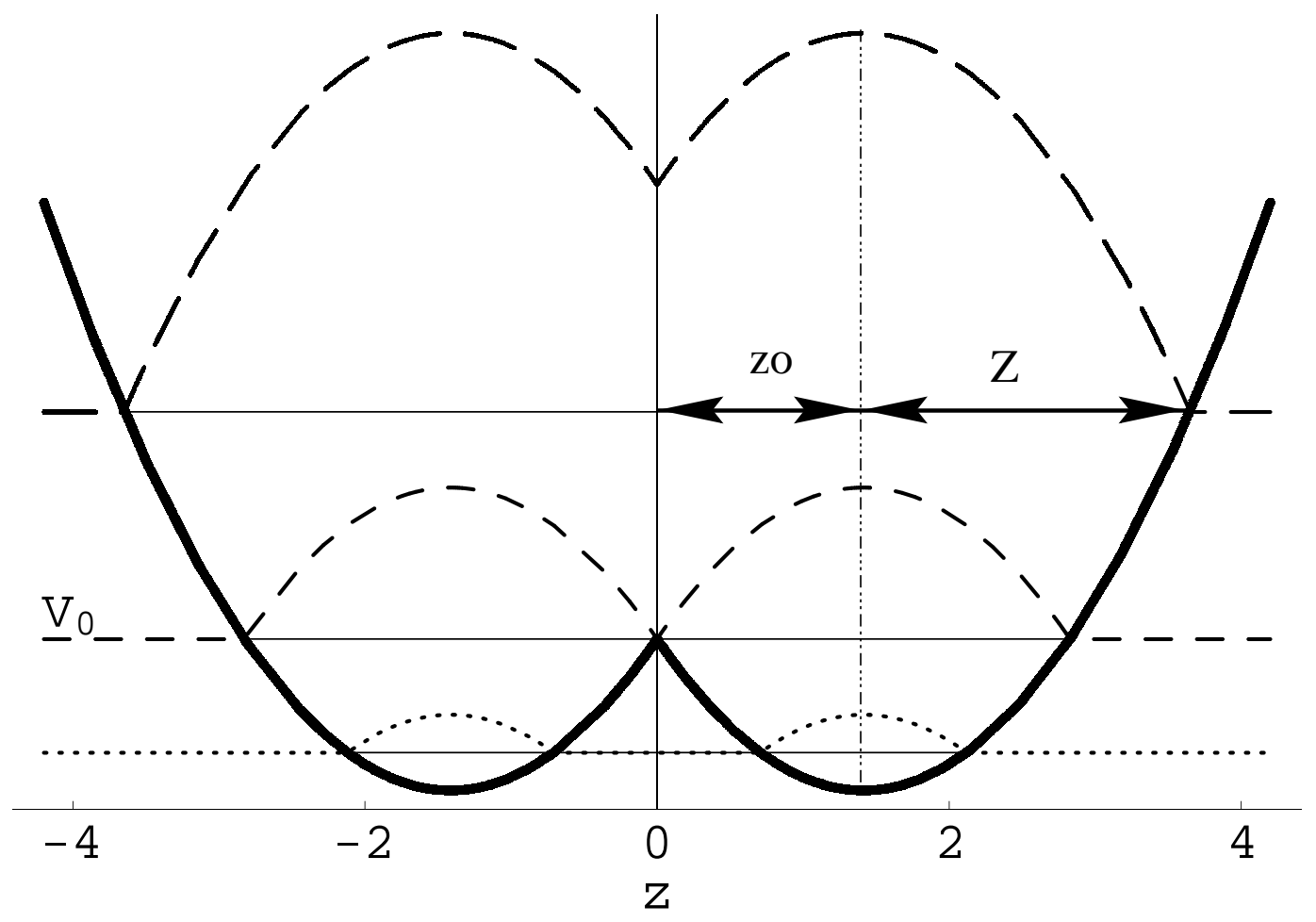

Figure 2. Capuzzi \& Hernandez 


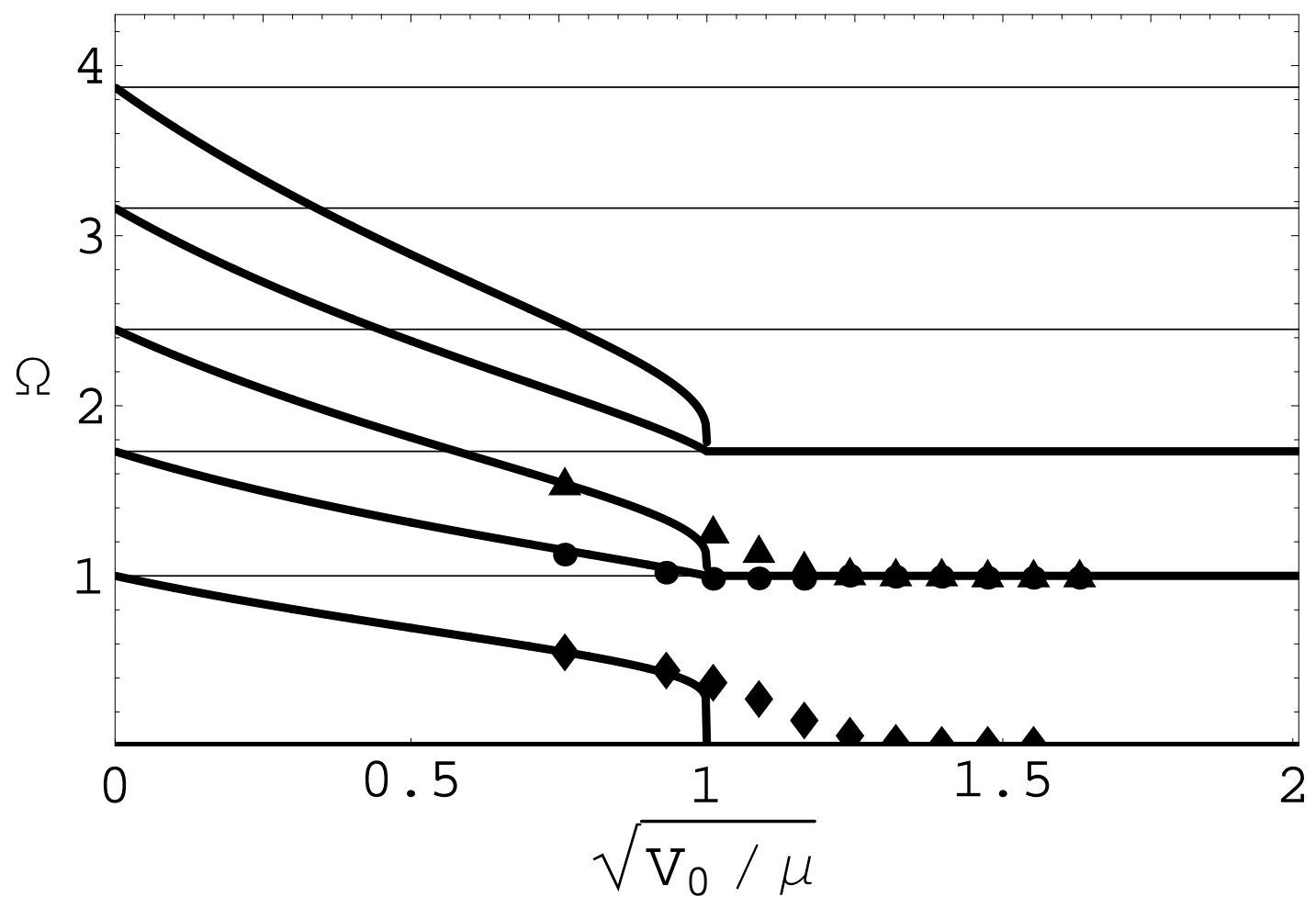

Figure 3. Capuzzi \& Hernandez 


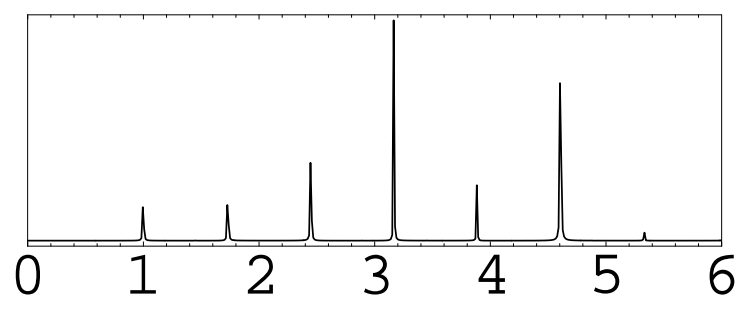

FFT
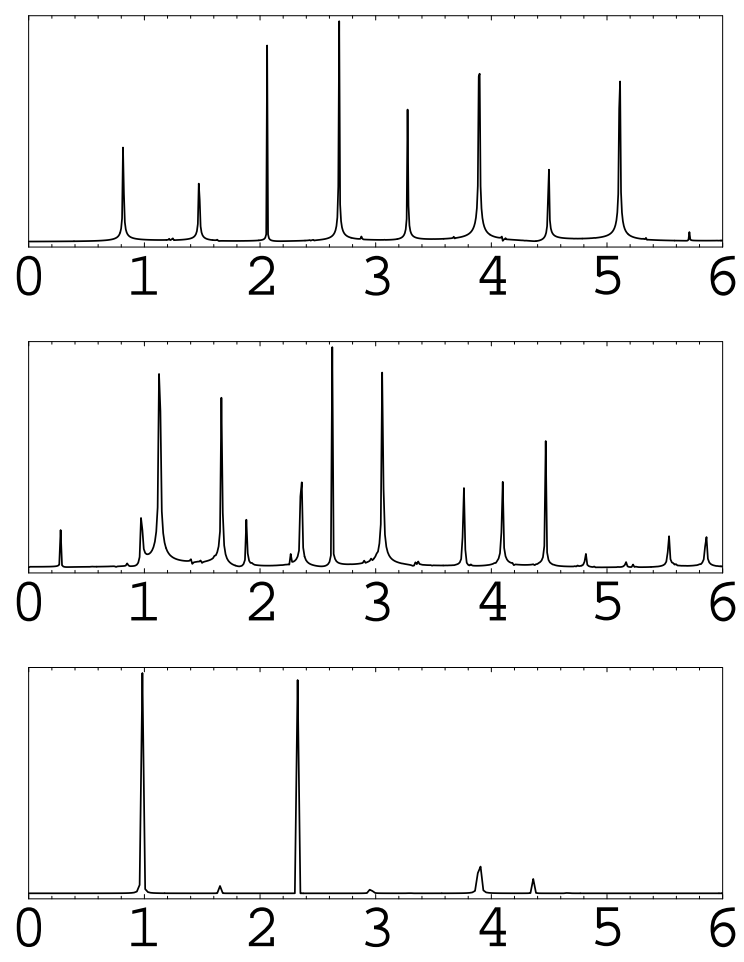

$\Omega$

Figure 4. Capuzzi \& Hernandez 


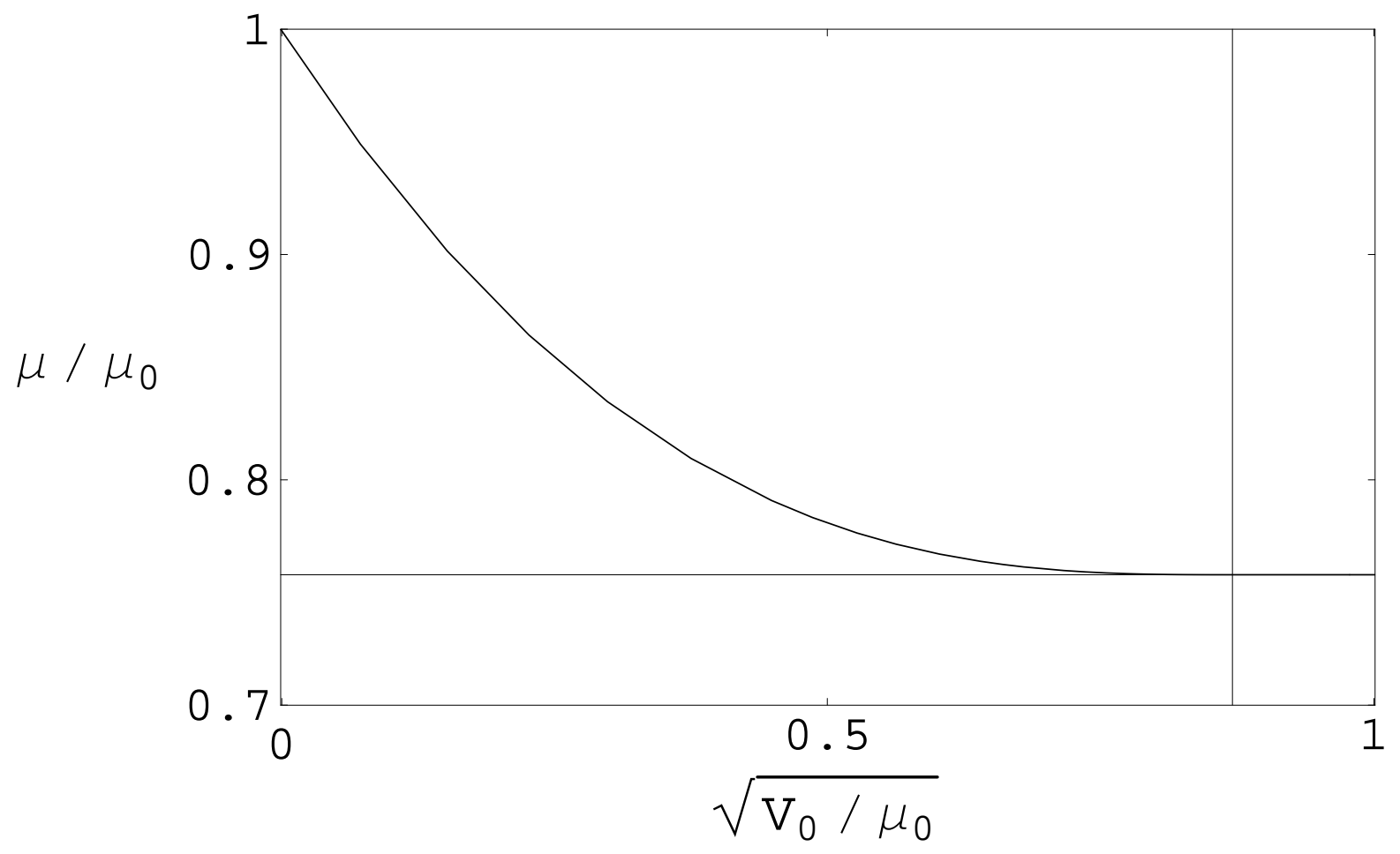

Figure 5. Capuzzi \& Hernandez 


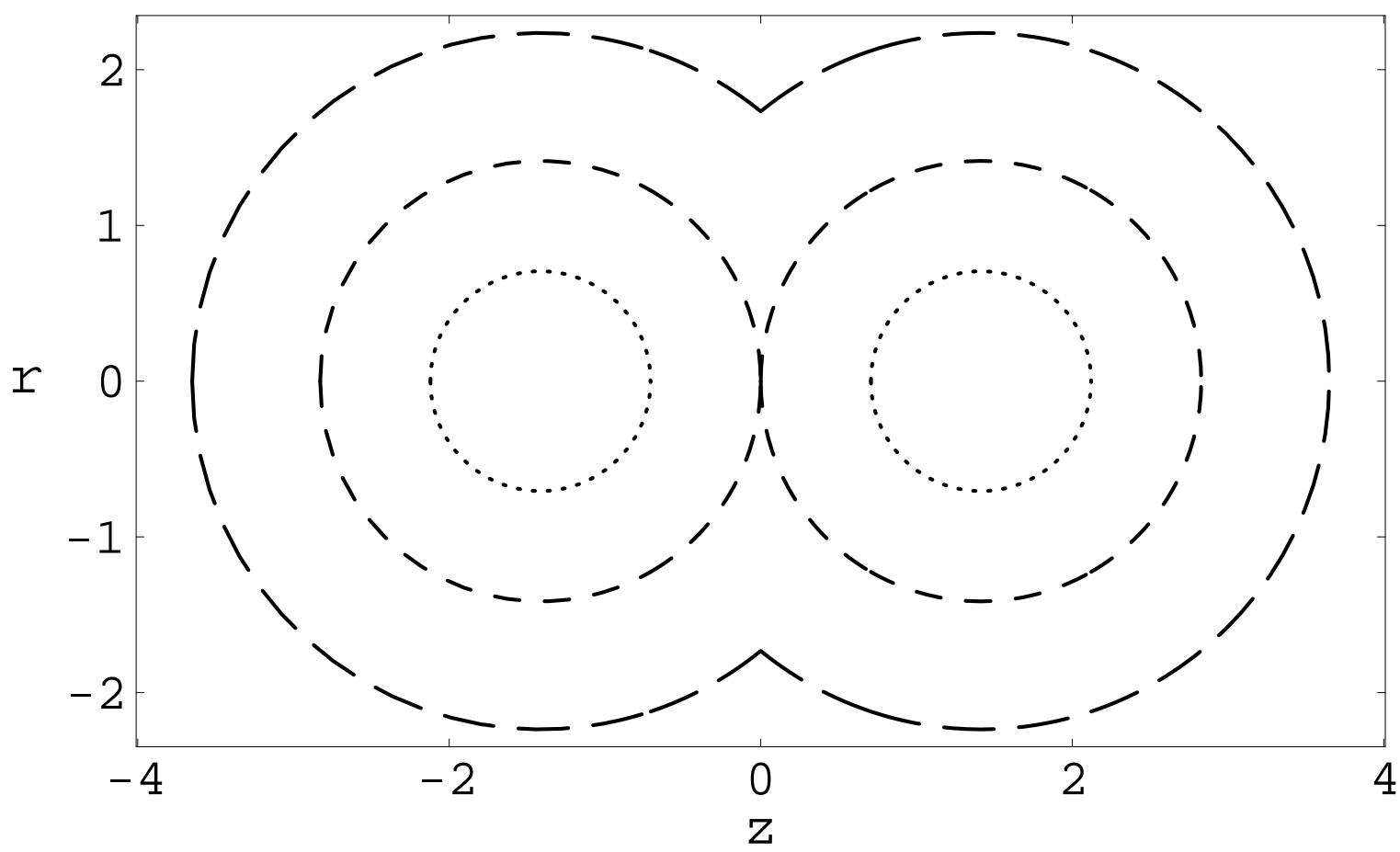

Figure 6. Capuzzi \& Hernandez 


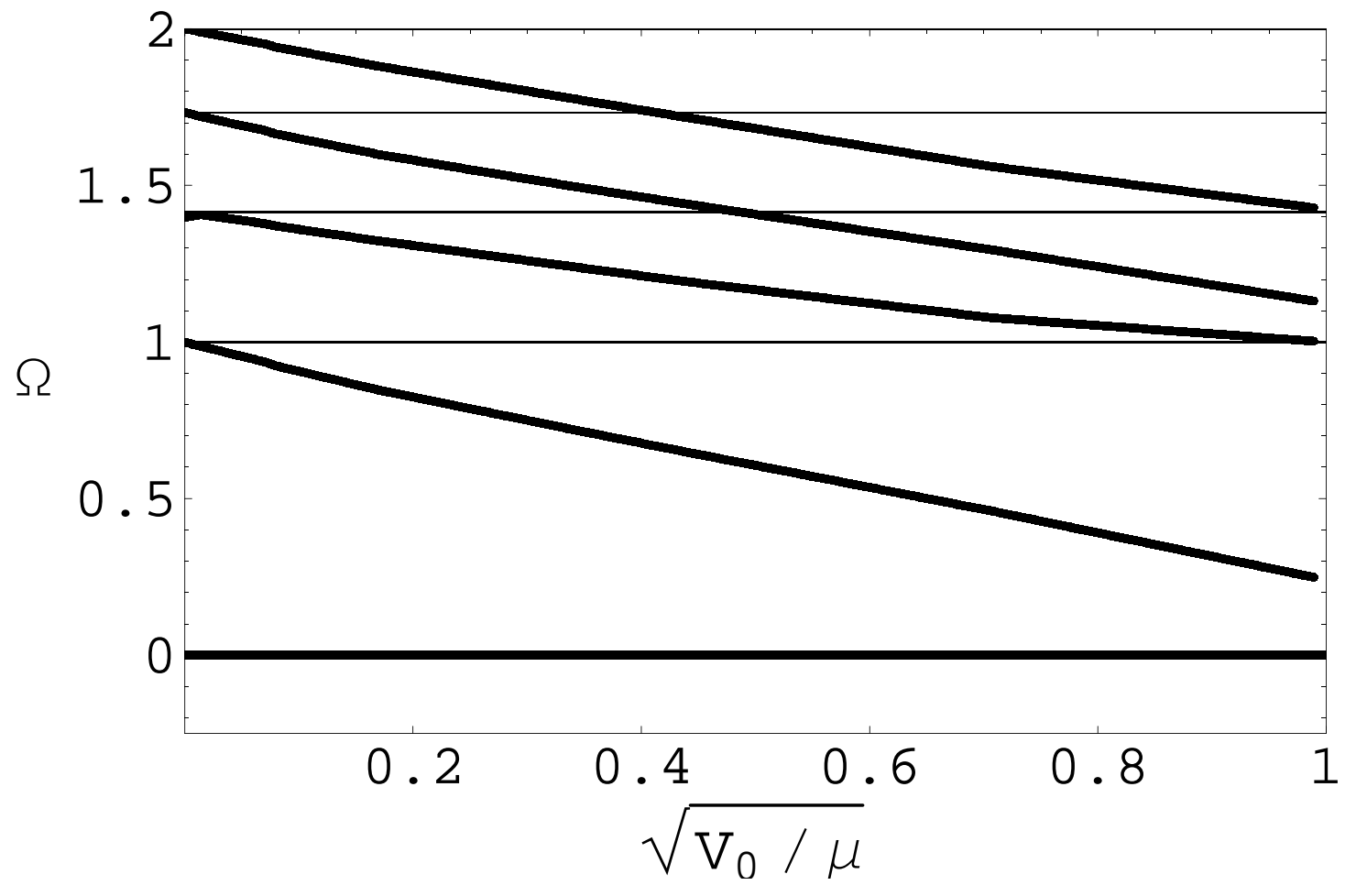

Figure 7a. Capuzzi \& Hernandez 


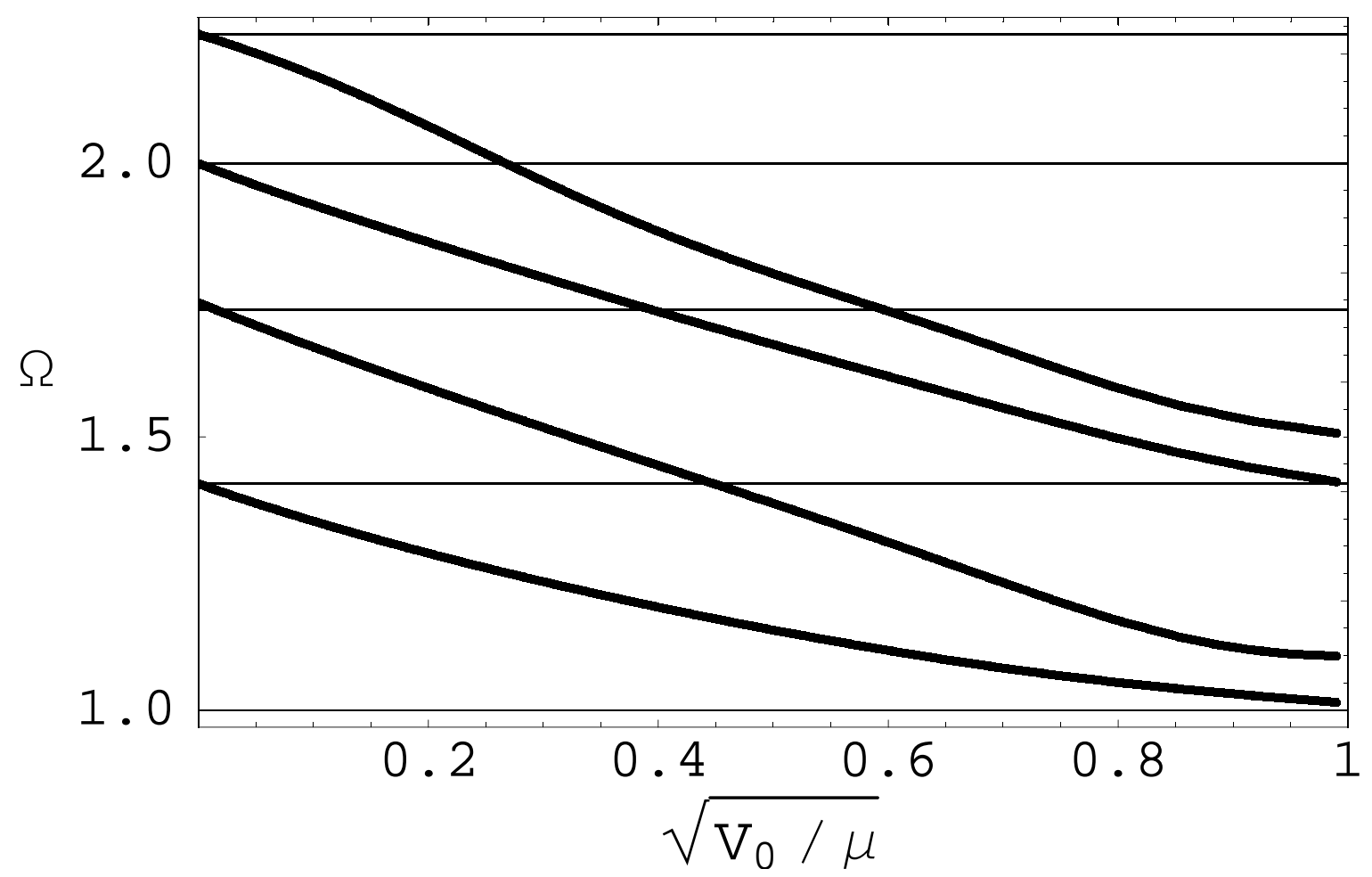

Figure 7b. Capuzzi \& Hernandez 


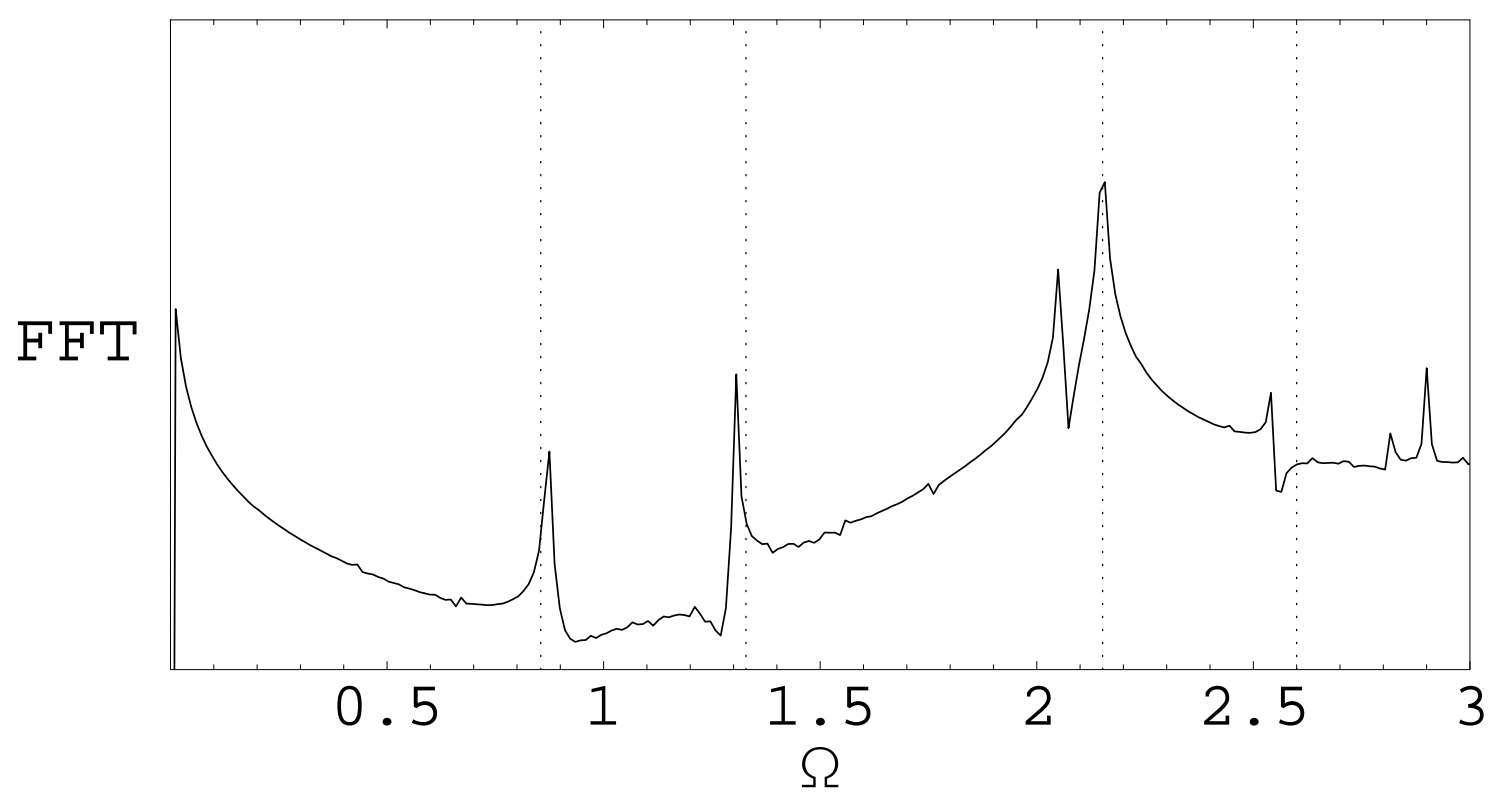

Figure 8. Capuzzi \& Hernandez 

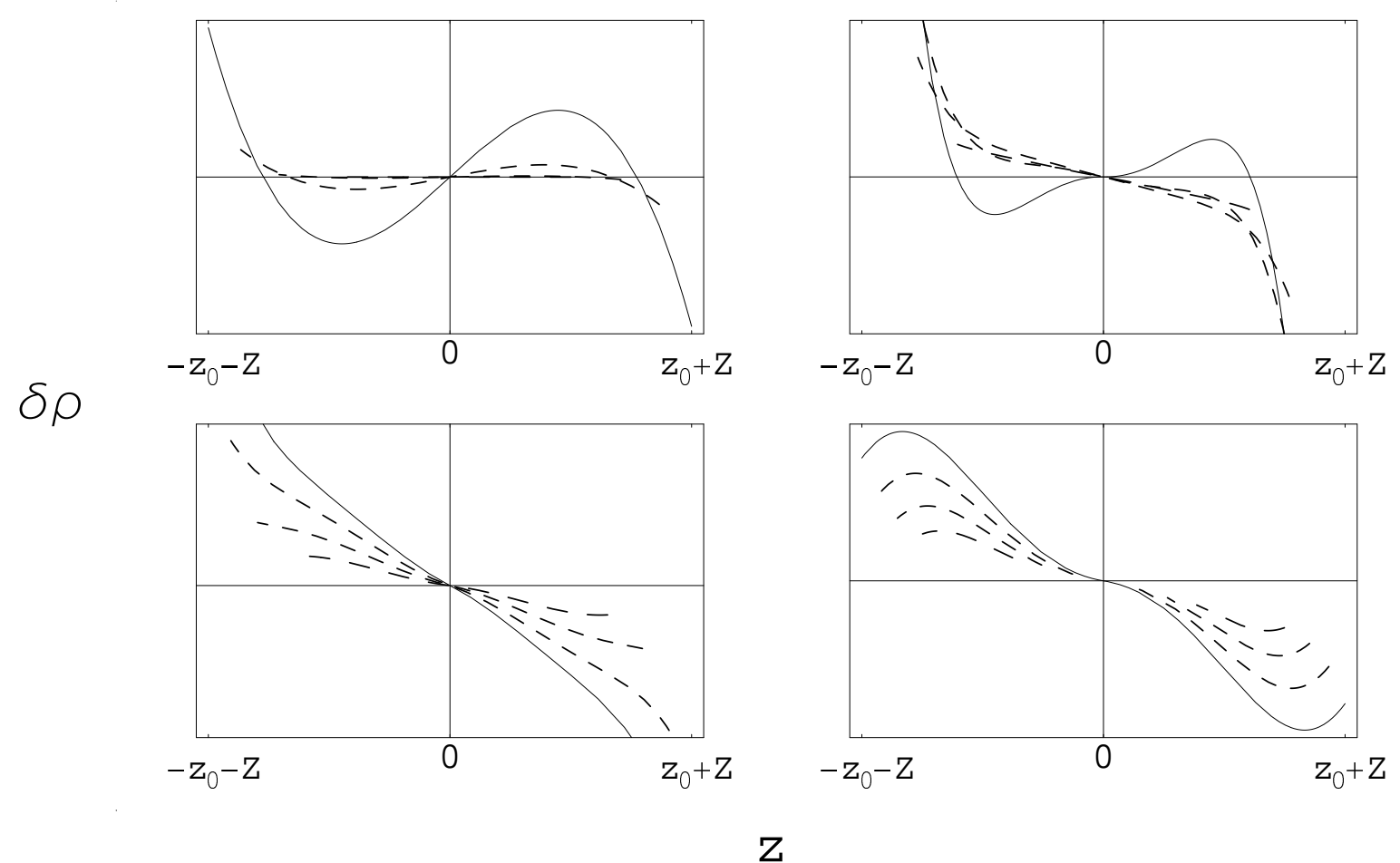

Figure 9. Capuzzi \& Hernandez 

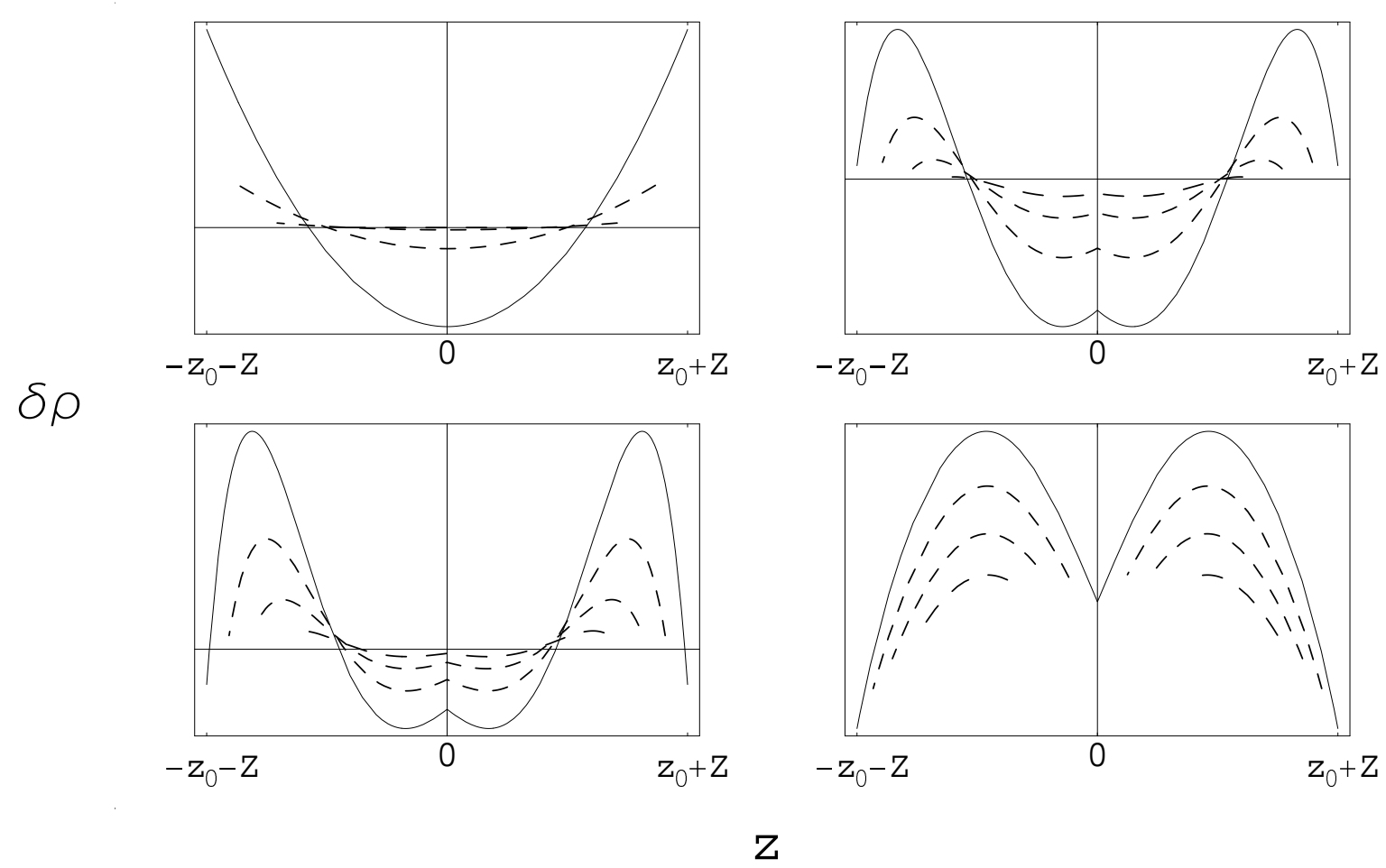

Figure 10. Capuzzi \& Hernandez 

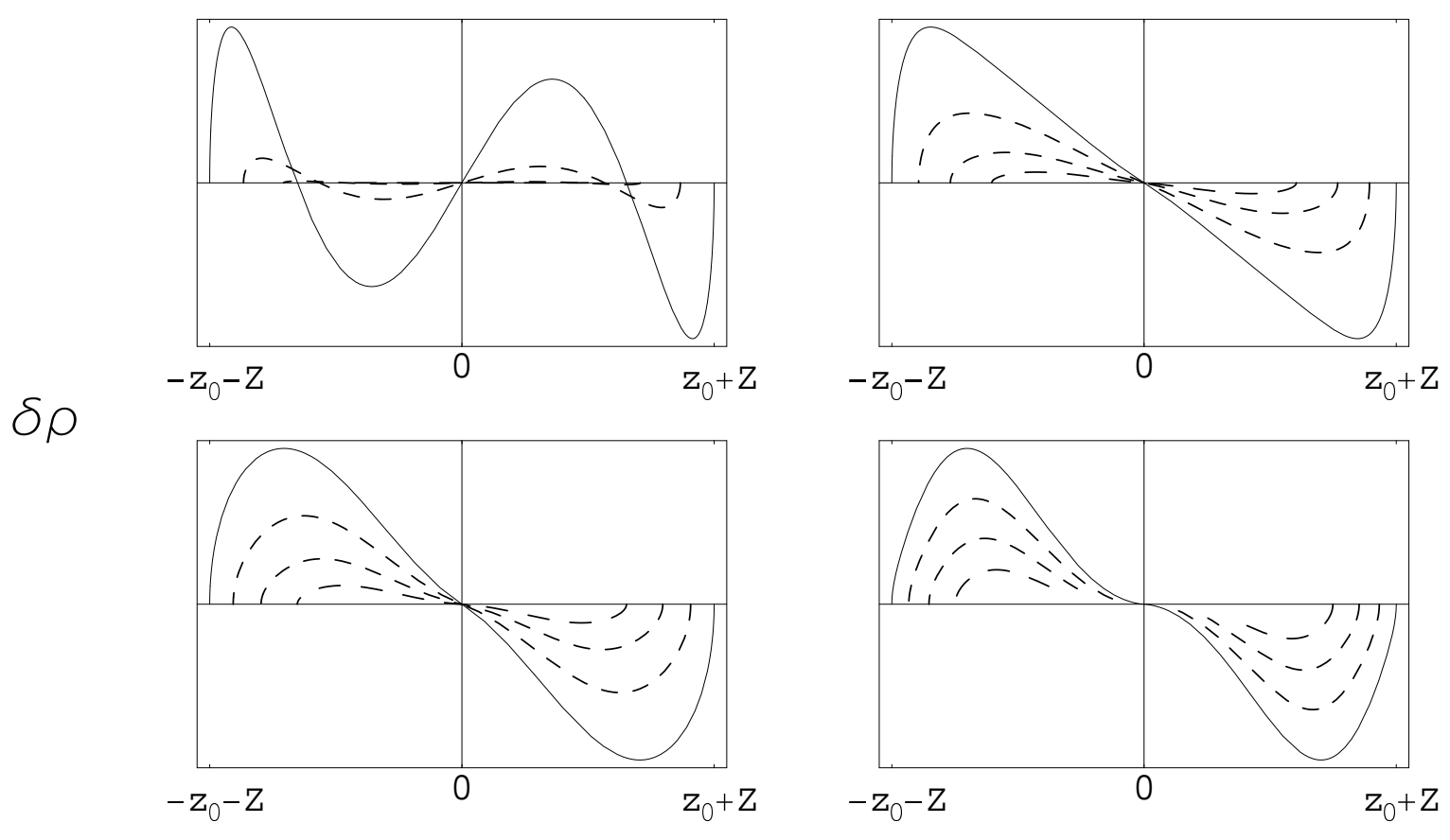

Z

Figure 11. Capuzzi \& Hernandez 

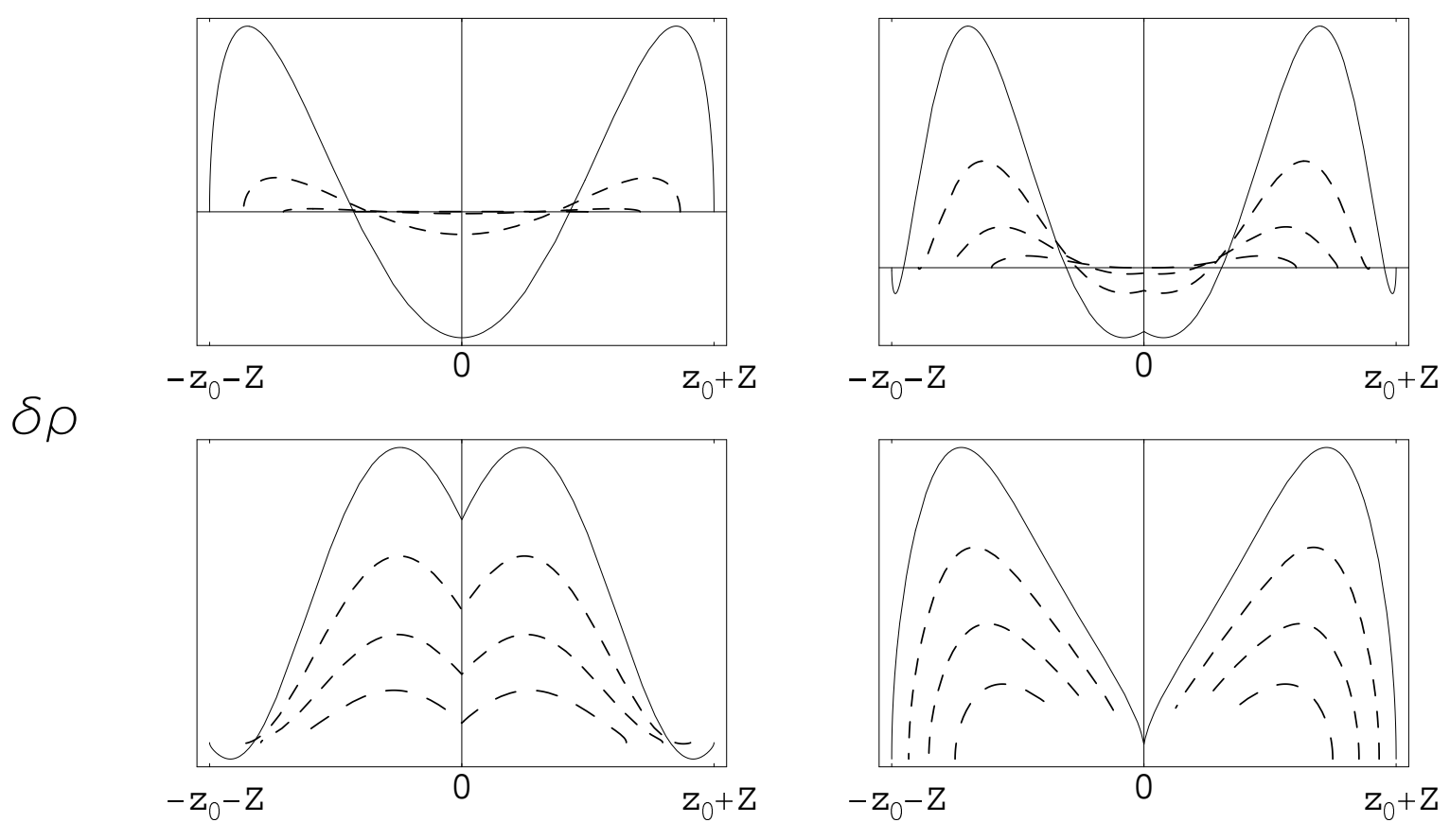

$\mathrm{Z}$

Figure 12. Capuzzi \& Hernandez 